\title{
Preventing Multipartite Disentanglement by Local Modulations
}

\author{
G. Gordon and G. Kurizk $*$ \\ Department of Chemical Physics, Weizmann Institute of Science, Rehovot 76100, Israel
}

(Dated: September 2, 2018)

\begin{abstract}
An entangled multipartite system coupled to a zero-temperature bath undergoes rapid disentanglement in many realistic scenarios, due to local, symmetry-breaking, differences in the particle-bath couplings. We show that locally controlled perturbations, addressing each particle individually, can impose a symmetry, and thus allow the existence of decoherence-free multipartite entangled systems in zero-temperature environments.
\end{abstract}

PACS numbers: 03.65.Yz, 03.65.Ta, 42.25.Kb

Symmetry is a powerful means of protecting entangled quantum states against decoherence [1], since it allows the existence of a decoherence-free subspace or a decoherence-free subsystem (DFS) 2, 3]. In multipartite systems, this requires that all particles be perturbed by the same environment. In keeping with this requirement, quantum communication protocols based on entangled two-photon states have been studied under collective depolarization conditions, namely, identical random fluctuations of the polarization for both photons [4]. Entangled qubits that reside at the same site or at equivalent sites of the system, e.g. atoms in optical lattices, have likewise been assumed to undergo identical decoherence [1].

However, locally-decohering entangled states of two or more particles, such that each particle travels along a different channel or is stored at a different site in the system, may break this symmetry. A possible consequence of this symmetry breaking is the abrupt "death" of the entanglement [5]. Such systems, composed of particles undergoing individual or "local" decoherence, do not possess a natural DFS and thus present more challenging problems insofar as decoherence effects are concerned 6]. Specifically, can the multipartite symmetry, broken by "local" decoherence, be dynamically restored to allow a DFS? Alternatively, can we dynamically impose other symmetries that protect all particles, not just part of them, from local decoherence? Previous treatments [3] have suggested to dynamically restore a DFS by subjecting the particles to sufficiently frequent, fast and strong pulses, assuming that they stroboscopically decouple all particles from the bath, irrespective of their coupling strengths.

Here we address these fundamental questions by developing a generalized treatment of multipartite entangled states (MES) decaying into zero-temperature baths and subject to arbitrary external perturbations whose role is to provide dynamical protection from decay and decoherence. Our treatment applies to any difference between the couplings of individual particles to the baths. It does not assume the perturbations to be stroboscopic, i.e. strong or fast enough, but rather to act concurrently with the particle-bath interactions. Our main results are

${ }^{*}$ Electronic address: gershon.kurizki@weizmann.ac.il to show that by applying local (selective) perturbations to multilevel particles, i.e. by addressing each level and each particle individually, one can create a decoherencefree system of many entangled qubits. Alternatively, one may reduce the problem of locally decohering MES to that of a single decohering particle, whose dynamical control has been thoroughly investigated [7, 8, [9].

In our treatment we introduce the multipartite system and arrive at a general dynamical solution for its decoherence. We then analyze realizations of different symmetries of the decoherence matrix comparing the resulting fidelities of the decohering state. Our system comprises $N$ particles, labelled by $j=1, \ldots, N$, each having a ground state $|g\rangle_{j}$ and $M_{j}$ excited states, $\left\{|n\rangle_{j}\right\}$ with energies, $\hbar \omega_{j, n}$. In particular, $n_{j}$ may enumerate the motional states of a trapped ion or atom, [6]. The coupling to the zero-temperature bath may differ from one particle to another and from one excited level to another. For their protection from decay and decoherence, we apply a perturbation such that each level is modulated by a different $A C$ Stark shift $\hbar \delta_{j, n}(t)$ and/or by different off-resonant perturbing field $\tilde{\epsilon}_{j, n}(t)$. The total Hamiltonian is the sum of the multipartite system, bath and off-diagonal system-bath interaction Hamiltonians [10]:

$$
\begin{aligned}
& H(t)=\hbar \sum_{j=1}^{N} \sum_{n=1}^{M_{j}}\left[\omega_{j, n}+\delta_{j, n}(t)\right]|n\rangle_{j}\left\langle n\left|+\hbar \sum_{k} \omega_{k}\right| k\right\rangle\langle k|+ \\
& \hbar \sum_{j=1}^{N} \sum_{k} \sum_{n=1}^{M_{j}}\left[\tilde{\epsilon}_{j, n}(t) \mu_{k, j, n}(|n\rangle\langle g|)_{j}|v a c\rangle\langle k| \otimes I_{j^{\prime} \neq j}+\text { H.c. }(1)\right.
\end{aligned}
$$

Here $I$ is the identity operator, $\mu_{k, j, n}$ is the off-diagonal coupling coefficient of the $n^{\text {th }}$ excited level of particle $j$ to an excitation of the bath mode $|k\rangle$ and $|v a c\rangle$ is the vacuum state of the bath. H.c. are Hermitian conjugates.

Although our strategy applies in general to any number of excitations, simple closed-form solutions are obtainable for a single initial excitation of the system. Accordingly, the complete wave function is:

$$
|\Psi(t)\rangle=\sum_{k} \alpha_{0}^{k}(t)|k\rangle \bigotimes_{j=1}^{N}|g\rangle_{j}+\sum_{j=1}^{N} \sum_{n=1}^{M_{j}} \alpha_{j, n}(t)|n\rangle_{j}|v a c\rangle \bigotimes_{j^{\prime} \neq j}|g\rangle_{j^{\prime}}
$$


We will denote the first and second RHS terms as the bath and system wave function, $\left|\Psi^{B}(t)\right\rangle$ and $\left|\Psi^{S}(t)\right\rangle$, respectively. In order to solve the Schrödinger equation, one may eliminate the $\left\{\alpha_{0}^{k}(t)\right\}$ amplitudes and transform to the interaction picture, ending up with an exact integro-differential equation. Assuming that these amplitudes are slowly varying on the time-scale of the bath response [8] and using the matrix representation, this equation has the general solution:

$$
\tilde{\boldsymbol{\alpha}}(t)=\mathrm{T}_{+} e^{-\boldsymbol{J}\left(t^{\prime}\right)} \tilde{\boldsymbol{\alpha}}(0), \quad \tilde{\alpha}_{j, n}=e^{i \omega_{j, n} t+i \int_{0}^{t} d \tau \delta_{j, n}(\tau)} \alpha_{j, n} .
$$

where $\tilde{\boldsymbol{\alpha}}=\left\{\tilde{\alpha}_{j, n}\right\}, \mathrm{T}_{+}$is the time-ordering operator and $\boldsymbol{J}(t)=\left\{J_{j j^{\prime}, n n^{\prime}}(t)\right\}$ is the dynamically-modified decoherence matrix, determined by the following convolution:

$$
\begin{aligned}
& J_{j j^{\prime}, n n^{\prime}}(t)=2 \pi \int_{-\infty}^{\infty} d \omega G_{j j^{\prime}, n n^{\prime}}(\omega) K_{t, j j^{\prime}, n n^{\prime}}(\omega) \\
& G_{j j^{\prime}, n n^{\prime}}(\omega)=\hbar^{-2} \sum_{k} \mu_{k, j, n} \mu_{k, j^{\prime}, n^{\prime}}^{*} \delta\left(\omega-\omega_{k}\right) \\
& K_{t, j j^{\prime}, n^{\prime \prime}}(\omega)=\epsilon_{t, j, n}^{*}\left(\omega-\omega_{j, n}\right) \epsilon_{t, j^{\prime}, n^{\prime}}\left(\omega-\omega_{j^{\prime} n^{\prime}}\right) \\
& \epsilon_{t, j, n}(\omega)=\int_{0}^{t} d \tau \epsilon_{j, n}(\tau) e^{i \omega \tau} \\
& \epsilon_{j, n}(t)=\tilde{\epsilon}_{j, n}(t) e^{-i \int_{0}^{t} d \tau \delta_{j, n}(\tau)}
\end{aligned}
$$

Here $G_{j j^{\prime}, n n^{\prime}}(\omega)$ is the coupling spectrum matrix given by nature and $K_{t, j j^{\prime}, n n^{\prime}}(\omega)$ is the dynamical modulation matrix, which we design at will to suppress the decoherence (cf. (10). This general solution holds for dynamically-modified relaxation of a singly-excited MES into a zero-temperature bath.

For example, we may control the decoherence by impulsive phase modulation, i.e. a sequence of pulsed Stark shifts caused by fields whose amplitudes satisfy [8]

$$
\begin{aligned}
& \epsilon_{j, n}(t)=e^{i\left[t / \tau_{j, n}\right] \theta_{j, n}} \\
& \epsilon_{t, j, n}(\omega)=\frac{\left(e^{i \omega \tau_{j, n}}-1\right)\left(e^{i\left(\theta_{j, n}+\omega \tau_{j, n}\right)\left[t / \tau_{j, n}\right]}-1\right)}{i \omega\left(e^{i\left(\theta_{j, n}+\omega \tau_{j, n}\right)}-1\right)}(10)
\end{aligned}
$$

Here [...] denote the integer part, $\tau_{j, n}$ and $\theta_{j, n}$ are the pulse duration and the phase change for level $n$ of particle $j$, respectively. In the limit of weak pulses, of area $\left|\theta_{j, n}\right| \ll \pi$, Eq. (10) yields $\epsilon_{t, j, n}(\omega) \cong \epsilon_{t, j, n} \delta\left(\omega-\Delta_{j, n}\right)$, where $\Delta_{j, n}=\theta_{j, n} / \tau_{j, n}$ is the effective spectral shift caused by the pulses.

It is expedient to rewrite the fidelity of the evolving system, $F(t)=\left|\left\langle\Psi^{S}(0) \mid \Psi^{S}(t)\right\rangle\right|^{2}$, as a product of two factors:

$$
\begin{aligned}
& F(t)=F_{p}(t) F_{c}(t) \\
& F_{p}(t)=|A(t)|^{2}=\sum_{j=1}^{N} \sum_{n=1}^{M_{j}}\left|\alpha_{j, n}(t)\right|^{2} \\
& F_{c}(t)=\frac{\left|\sum_{j=1}^{N} \sum_{n=1}^{M_{j}} \alpha_{j, n}^{*}(0) \alpha_{j, n}(t)\right|^{2}}{|A(t)|^{2}}
\end{aligned}
$$

where $F_{c}(t)$ is the autocorrelation function, $\left|\left\langle\Psi^{S}(0) \mid \Psi^{S}(t)\right\rangle\right|^{2}$, normalized by the total excitation probability $|A(t)|^{2}$. Thus $1-F_{p}(t)$ measures population loss from any $|n\rangle_{j}$, whereas $1-F_{c}(t)$ is a measure of correlation preservation: $F_{c}=1$ when the initial multipartite correlations are completely preserved. As shown below population and correlation preservation can be independently controlled in the model of Eqs. (11),(2).

In the absence of dynamical control, $F_{c}(t)$ decays much faster than $F_{p}(t)$ and is much more sensitive to the asymmetry between local particle-bath couplings. Thus, for initial Bell singlet and triplet states, $|\Psi(0)\rangle=$ $1 / \sqrt{2}\left(|g\rangle_{A}|n\rangle_{B} \pm|n\rangle_{a}|g\rangle_{B}\right)$, which do not experience cross-decoherence but only different local decoherence rates, $\alpha_{A(B)}(t)=1 / \sqrt{2} e^{-J_{A(B)}}(t)$. We find $F_{p}(t)=$ $\left(e^{-2 J_{A}(t)}+e^{-2 J_{B}(t)}\right) / 2 ; F_{C}(t)=(1+C(t)) / 2=1 / 2+$ $e^{-\Delta J(t)} /\left(1+e^{-2 \Delta J(t)}\right)$, where $\Delta J(t)=J_{A}(t)-J_{B}(t)$ and $C(t)$ is the concurrence [11].

We shall first deal with $N$ identical qubits, and thus ignore the $n$ subscript, i.e. set $\omega_{j} \equiv \omega_{0}$. We also require that at any chosen time $t=T$, the AC Stark shifts in Eq. (3) satisfy, $\int_{0}^{T} d \tau \delta_{j}(\tau)=2 \pi m$, where $m=0, \pm 1, \ldots$. This requirement ensures that modulations only affect the decoherence matrix (4), but do not change the relative phases of the entangled qubits when their MES is probed or manipulated by logic operations at $t=T$.

Without any modulations, decoherence in this scenario has no inherent symmetry. Our point is that one can symmetrize the decoherence by appropriate modulations. The key is that different, "local", phase-locked modulations applied to the individual particles, according to Eq. (6), can be chosen to cause controlled interference and/or spectral shifts between the particles' couplings to the bath. The $K_{t, j j^{\prime}}(\omega)$ matrices (cf.([6)) can then satisfy $2 N$ requirements at all times and be tailored to impose the advantageous symmetries described below. By contrast, a "global" (identical) modulation, characterized by $K_{t, j j^{\prime}}(\omega)=\left|\epsilon_{t}(\omega)\right|^{2}$, is not guaranteed to satisfy $N \gg 1$ symmetrizing requirements at all times (Fig. 1 $\mathrm{a}$ ). The most desirable symmetry is that of identical coupled particles (ICP), which would emerge if all the modulated particles could acquire the same dynamically modified decoherence and cross-decoherence yielding the following $N \times N$ fully symmetrized decoherence matrix

$$
J_{j j^{\prime}}^{\mathrm{ICP}}(t)=r(t) \quad \forall j, j^{\prime} .
$$

ICP would then give rise to a $(N-1)$-dimensional decoherence-free subspace: the entire single-excitation sector less the totally symmetric entangled state. An initial state in this DFS [2] would have $F(t)=1$ for all times, meaning that it would neither lose its population nor its initial correlations (or entanglement).

However, it is generally impossible to ensure this symmetry, since it amounts to satisfying $N(N-1) / 2$ conditions using $N$ modulating fields. Even if we accidently succeed with $N$ particles, the success is not scalable to $N+1$ or more particles. Moreover, the ability to impose 

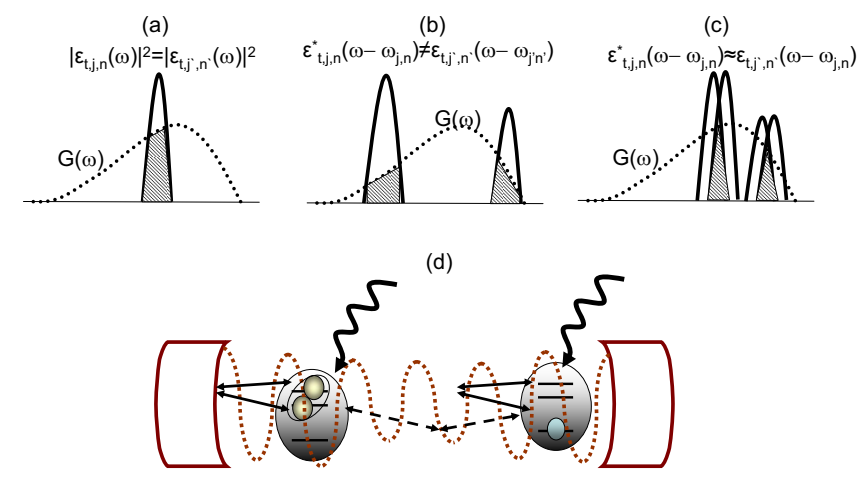

FIG. 1: Two 3 - level particles in a cavity, coupled to the cavity modes (thin lines) and subject to local control fields (thick lines). (a-c) Frequency domain overlap of coupling spectrum (dotted) and modulation matrix elements(solid), resulting in modified decoherence matrix elements (shaded), for: (a) global modulation, (b) cross-decoherence elimination (IIP symmetry) and (c) IIT symmetry. (d) IIT symmetry scheme.

the ICP symmetry by local modulation fails completely if not all particles are coupled to all other particles through the bath, i.e. if some $G_{j j^{\prime}}(\omega)$ elements vanish.

A more limited symmetry that we may ensure for $N$ qubits is that of independent identical particles (IIP). This symmetry is formed when spectral shifts and/or interferences imposed by $N$ modulations cause the $N$ different particles to acquire the same single-particle decoherence $r(t)$ and experience no cross-decoherence. To this end, we may choose in Eq. (91) $\epsilon_{t, j}(\omega) \simeq \epsilon_{t, j} \delta\left(\omega-\Delta_{j}\right)$. The spectral shifts $\Delta_{j}$ can be different enough to couple each particle to a different spectral range of bath modes so that their cross-coupling vanishes:

$$
J_{j j^{\prime}}(t)=\epsilon_{t, j}^{*} \epsilon_{t, j^{\prime}} \int d \omega G(\omega) \delta\left(\omega-\Delta_{j}\right) \delta\left(\omega-\Delta_{j^{\prime}}\right) \rightarrow 0 .
$$

Here, the vanishing of $G_{j j^{\prime}}(\omega)$ for some $j, j^{\prime}$ is not a limitation. The $N$ single-particle decoherence rates can be equated by an appropriate choice of $N$ parameters $\left\{\Delta_{j}\right\}$ :

$$
J_{j j^{\prime}}^{\mathrm{IIP}}(t)=\left|\epsilon_{t, j}\right|^{2} G_{j j}\left(\Delta_{j}\right)=\delta_{j j^{\prime}} r(t),
$$

where $\delta_{j j^{\prime}}$ is Kronecker's delta (Fig. 目). The IIP symmetry results in complete correlation preservation, i.e. $F_{c}(t)=1$, but still permits population loss, $F(t)=$ $F_{p}(t)=e^{-2 \operatorname{Rer}(t)}$. If the single-particle $r(t)$ may be dynamically suppressed, i.e. if the spectrally shifted bath response $G_{j j}\left(\omega_{j}+\Delta_{j}\right)$ is small enough, this $F(t)$ will be kept close to 1 (Fig. 22). If IIP symmetry is imposed, the particles become effectively independent in terms of their coupling to the bath. Hence, collective coupling to the bath, which is a prerequisite for a DFS, is not formed in this case. In order to impose decoherence-free conditions, we extend the treatment to multilevel particles [9]. We will show that the use of auxiliary levels and local modulations can result in a decoherence-free singly-excited $N$ qubit system, in a general and realistic coupling scenario.

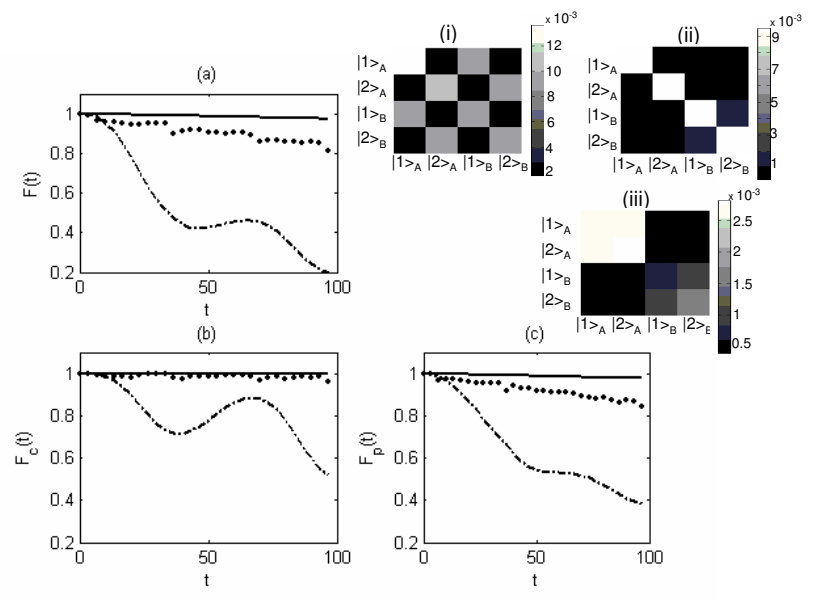

FIG. 2: Fidelity as a function of time (in units of $(10 \gamma)^{-1}$ ): (a) overall fidelity, (b) correlation preservation, and (c) population preservation. (i) Global $\pi$-phase flips impose no symmetry (dashed) $\left(\tau_{j, n}=1.1, \theta_{j, n} / \pi=1.0\right)$. (ii) independent identical particles (IIP) symmetry (dotted) $\left(\tau_{j, n}=\right.$ $\left.(0.75,0.85,0.95,1.05), \theta_{j, n} / \pi=(0.834,0.806,0.836,0.82)\right)$; (iii) independent identical trapping (IIT) symmetry $($ solid $)\left(\tau_{j, n}=(0.85,0.85,1.05,1.05), \theta_{j, n} / \pi=\right.$ $(0.924,0.9,0.945,0.91))$. Top-right: Decoherence matrix elements at time $t=100$. The parameters are: $\omega_{1}=0.5, \omega_{2}=0.6, \gamma=0.1, \quad k_{0} r_{\min }=1.0$, $t_{j, n}=(0.7,1.0,1.06,1.1), r_{j}=(0.0,0.1), \eta_{n} / \pi=(0.0,0.1)$.

To this end we invoke three-level particles with excited states $|1\rangle$ and $|2\rangle$. In a single three-level particle, an external field can impose an intraparticle DFS if $|1\rangle$ and $|2\rangle$ decay at the same rate to the ground state $|0\rangle$; this DFS consists of $|0\rangle$ and the "dark" or trapping state [10], the anti-symmetric superposition of the two excited states $1 / \sqrt{2}(|1\rangle-|2\rangle)$. However, this intraparticle DFS would be destroyed in a system of $N$ three-level particles that are coupled to the bath, and/or experience crossdecoherence. In order to remedy this problem, let us consider local modulations acting differently on the two excited levels within each particle. Such modulations can be tailored to impose what may be dubbed "independent identical trapping" (IIT) symmetry. This means that all particles acquire identical trapping states and become (effectively) independent, without cross-decoherence. As discussed above, $N$ spectral shifts $\Delta_{j}$ determined by $\left|\epsilon_{t, j}(\omega)\right|^{2}$ suffice to eliminate cross-decoherence, Eq. (15). Under these conditions, $N$ destructively interfering pairs of local fields will cause trapping in each particle (Fig. 11; 11). This would result in an $N$-dimensional DFS, composed of $N$ particles sharing single-excitation with antisymmetrically superposed excited states. Under the IIT symmetry the decoherence matrix is block diagonal, each block corresponding to particle $j$ with levels $n, n^{\prime}$ :

$$
J_{j j^{\prime}, n n^{\prime}}^{\mathrm{IIT}}(t)=\delta_{j j^{\prime}} r_{j}(t)
$$

Both disentanglement and population loss are nearly 
completely eliminated within the multipartite subspace, resulting in $F(t)$ very close to 1 (Fig. [2).

As an example, the IIT recipe will be analyzed for two three-level particles, where each level of each particle experiences different coupling to the bath, and crossdecoherence exists. Let us take the initial state of particles $A, B$ to be $\left|\Psi_{S}(0)\right\rangle=\left(|-\rangle_{A}|0\rangle_{B} \pm|0\rangle_{A}|-\rangle_{B}\right) / \sqrt{2}$, where $(j=A, B)|-\rangle_{j}=\left(|1\rangle_{j}-|2\rangle_{j}\right) / \sqrt{2}$. The goal will be to keep this state intact, $\left|\Psi_{S}(t)\right\rangle \simeq\left|\Psi_{S}(0)\right\rangle$, by preventing cross-decoherence and imposing intraparticle destructive interference. The bath response matrix will be taken to be $\Phi_{j j^{\prime}, n n^{\prime}}(t)=\gamma d_{n} d_{n^{\prime}} \frac{e^{-t^{2} / 4 t_{j, n}^{2}} e^{-t^{2} / 4 t_{j^{\prime}, n^{\prime}}^{2}}}{k_{0}\left(r_{m i n}+r_{j j^{\prime}}\right)}$ where $\gamma$ is the single-particle unperturbed decay rate (satisfying Fermi's Golden Rule 10]), $d_{n}=\cos \eta_{n}$, with $\eta_{n}$ being the angle of transition dipole, $t_{j, n}$ is the correlation time of level $n$ of particle $j, k_{0}$ is the wavevector of the resonant transition $|0\rangle \leftrightarrow|1(2)\rangle, r_{\text {min }}$ is the distance below which the particles are identically coupled to the bath and $r_{j j^{\prime}}=\left|\boldsymbol{r}_{j}-\boldsymbol{r}_{j^{\prime}}\right|$, where $\boldsymbol{r}_{j}$ is the position of particle $j$. This model may describe the distance-dependent cross-decoherence of either radiatively or vibrationally relaxing atoms or ions at different sites in a trap, lattice or cavity [12, 13]. The parameters of the pulse sequence in Eq. (9) for creating the IIP or IIT symmetries were chosen such that the faster decaying particle experienced the stronger and/or faster pulses, i.e. $\theta_{j, n} / \tau_{j, n}$ were adapted to the bath response parameters $\Phi_{j j, n n}$ of each particle. The pulse sequences were chosen to be different enough for each particle in order to eliminate the desired crossdecoherence terms, as per Eq. 151. Individual pulses were selected to obey the other IIP or IIT requirements described above. The very high fidelity achievable by IIT is seen in Fig. 2]
We may apply these considerations, for example, to cold ions or atoms in a cavity [13], whose radiative decoherence rates depend on their position within the cavity and may vary by as much as $15 \%$. This implies that placing several ions in the same cavity breaks their multipartite symmetry. AC Stark shifts are then an effective tool to restore the symmetry. To impose the IIP symmetry, one requires an impulsive phase modulation at a rate exceeding the cavity-mode linewidth, $1 / \tau \geq \Gamma$ (typically $\sim 12 \mathrm{GHz}$ ). In order to impose the IIT symmetry, one can either use two excited states of the ion, or two ions for each qubit, such that their singlet Bell state and the ground state form the DFS [14]. The modulations should be local, addressing each level and each ion separately, and adjusting the rate and phase of the impulsive phase modulation so as to impose the IIT multipartite symmetry, thus creating an $N$-qubit DFS. Recent experiments 13] indicate that such addressability is feasible either by spatial or spectral resolution.

To conclude, we have shown that local modulations are generally far more apt than identical (global) modulation to impose multipartite symmetry on an otherwise completely asymmetrically relaxing system. In particular, local modulations can impose the IIT symmetry, in a system of $N$ three-level particles, thus creating a decoherence-free singly-excited $N$-qubit system. The general formalism presented here spans the entire range of possible coupling scenarios and modulation schemes. These results imply that the challenge of multipartite decoherence, plaguing quantum information transmission and storage, may be successfully met in a variety of experimental situations.

We acknowledge the support of ISF and EC (QUACS and SCALA Networks).
[1] V. Akulin, A. Sarfati, G. Kurizki, and S. Pellegrin, eds., Decoherence, Entanglement and Information Protection in Complex Quantum Systems (Springer, 2005).

[2] P. Zanardi and M. Rasetti, Phys. Rev. Lett. 79, 3306 (1997); D. A. Lidar, I. L. Chuang, and K. B. Whaley, Phys. Rev. Lett. 81, 2594(1998); D. A. Lidar, D. Bacon, and K. B. Whaley, Phys. Rev. Lett. 82, 4556(1999); E. Knill, R. Laflamme, and L. Viola, Phys. Rev. Lett. 84, 2525(2000); P. Zanardi and S. Lloyd, Phys. Rev. Lett. 90, 067902 (2003); R. G. Unanyan, and M. Fleischhauer, Phys. Rev. Lett. 90, 133601(2003).

[3] L. Viola, E. Knill, and S. Lloyd, Phys. Rev. Lett. 85, 3520(2000); L. A. Wu, and D. A. Lidar, Phys. Rev. Lett. 88, 207902(2002); P. Facchi, and S. Pascazio, Phys. Rev. Lett. 89, 080401(2002); P. Facchi, D. A. Lidar, and S. Pascazio, Phys. Rev. A 69, 032314(2004); E. Brion el al., Phys. Rev. A 71, 052311(2005).

[4] K. Banaszek, A. Dragan, W. Wasilewski, and C. Radzewicz, Phys. Rev. Lett. 92, 257901 (2004); J.L. Ball, A. Dragan, and K. Banaszek, Phys. Rev. A. 69, 042324 (2004); L. Duan, M. Lukin, J. I. Cirac, and P. Zoller, Nature 414, 413 (2001); T. Y. Chen et al., quant-ph/0605206.

[5] T. Yu, and J. H. Eberly, Phys. Rev. Lett. 93, 140404 (2004); S. Bandyopadhyay, and D. A Lidar, Phys. Rev. A 70, 010301 (2004).

[6] A. D. Lisi and K. Mølmer, Phys. Rev. A 66, 052303 (2002); J. Sherson, and K. Mølmer, Phys. Rev. A 71, 033813 (2005).

[7] G. S. Agarwal, M. O. Scully, and H. Walther, Phys. Rev. A 63, 044101 (2001); Phys. Rev. Lett. 86, 4271 (2001); D. Vitali, and P. Tombesi, Phys. Rev. A 65, 012305 (2001).

[8] A. G. Kofman and G. Kurizki, Nature (London) 405, 546 (2000); Phys. Rev. Lett. 87, 270405 (2001); Phys. Rev. Lett. 93, 130406 (2004); A. Barone, G. Kurizki, and A. G. Kofman, Phys. Rev. Lett. 92, 200403 (2004).

[9] G. Gordon, G. Kurizki, and A.G. Kofman, J. Opt. B. 7, $283(2005)$.

[10] M. O. Scully and M. S. Zubairy, Quantum Optics (Cambridge University Press, Cambridge, 1997).

[11] W.K. Wootters, Phys. Rev. Lett. 80, 2245 (1998).

[12] S. Folling et al., Nature 434, 481 (2005).

[13] A. Kreuter et al., Phys. Rev. Lett. 92, 203002 (2004); 
D. Schrader et al., Phys. Rev. Lett. 93, 150501 (2004).

[14] D. Petrosyan and G. Kurizki, Phys. Rev. Lett. 89,

$207902(2002)$ 